\title{
Significance of Early Neuro-rehabilitation among Stroke Patients
}

\author{
Khurshid Mahmood \\ Professor, Department of Physical Medicine and Neurorehabilitation, National Institute of Neurosciences \& Hospital, \\ Dhaka, Bangladesh; Email: tannibabu@yahoo.com; Cell no.: +8801716854395
}

\begin{abstract}
A wide range of treatment techniques and approaches from different philosophical backgrounds are utilized in Neurological Rehabilitation. Research to support the different approaches varies hugely, with a wealth of research to support the use of some techniques while other approaches have limited evidence to support its use but rely on ancedotal evidence. The past decade has seen an exponential growth in the number of Randomized Control Trials in relation to physiotherapy interventions utilized in Stroke. The number of RCTs on Stroke Interventions has almost quadrupled in the past 10 years, with strong evidence seen in 30 out of 53 interventions for beneficial effects on one or more outcomes. They suggest the main change lies in the increased number of interventions to which 'strong evidence' could be assigned and an increase in the number of outcomes for which the findings are statistically significant.
\end{abstract}

Higher intensity of practice appears to be an important aspect of effective physical therapy and suggestion is that intensity of practice is a key factor in meaningful training after stroke, and that more practice is better. 17 hours of therapy over a 10 week period has been found to be necessary for significant positive effects at both the body function level as well as activities and participation level of the ICF. Yet despite the fact that National Clinical Guidelines advocate for at least 45 mins of therapy daily as long as there are rehabilitation goals and the patient tolerate this intensity, and recognition that high-intensity practice is better there still remains a big contrast between the recommended and actual applied therapy time. Recent surveys in the Netherlands show that patients with stroke admitted to a hospital stroke unit only received a mean of 22 minutes of physical therapy on weekdays. Similarly, in the United Kingdom inpatients received 30.6 minutes physical therapy per day. Both of these significantly fall short of the recommended 45 mins daily.

While the body of knowledge in relation to physiotherapy in stroke rehabilitation is still growing further confirmation of the evidence for physiotherapy after stroke, and facilitating the transfer to clinical practice, requires a better understanding of the neurophysiological mechanisms, including neuroplasticity, that drive stroke recovery, as well as the impact of physiotherapy interventions on these underlying mechanisms. Similarly further research is required to support physiotherapy implementation strategies in order to optimize the transfer of scientific knowledge into clinical practice. High growth in evidence does in its own way create challenges for physiotherapists in keeping up to date with new evidence as it becomes available and there is a need for further investigation into more effective and efficient methods for physiotherapists to keep their knowledge and skill level up-to-date in the long term.

Ability to change position and posture is affected in many individuals post stroke as a result of varying degrees of physical impairments. Therapeutic positioning aims to reduce skin damage, limb swelling, shoulder pain or subluxation, and discomfort, and maximize function and maintain soft tissue length. It is also suggested that positioning may assist in reduction of respiratory complications such as those caused by aspiration and avoid compromising hydration and nutrition. The aim of positioning the patient is to try to promote optimal recovery and comfort by modulating muscle tone, providing appropriate sensory information, increasing spatial awareness, improved ability to interact with the environment and prevention of complications such as pressure sores, and contracture.

The most appropriate position in which to place a patient following a stroke remains unclear. There is no Randomized Control Trial evidence to support the recommendation of any one position over another but five main positions have been recommended, a survey of physiotherapists' current positioning practices found the most commonly recommended positions to be sitting in an armchair as recommended by $98 \%$ of respondents; side lying on the unaffected side then side lying on the affected side. Sitting in a wheelchair and supine lying were less commonly recommended.

Immobility is associated with a number of post stroke complications such as deep vein thrombosis etc. Early mobilization aims to reduce the time that elapses between stroke and the first time the patient leaves the bed, increasing the amount of physical activity that the patient engages in outside of bed. Early mobilization 
like activities such as sitting out of bed, transfers, Therefore, post stroke physiotherapy is very essential standing and walking aims to minimize the risk of the complications of immobility and improve functional recovery. Early mobilization is defined as "mobilizing a patient out of bed within 24 hours after the stroke and encouraging them to practice outside the bed'. for early recovery of the patients.

[Journal of National Institute of Neurosciences Bangladesh, 2018;4(1):1-2] 